\title{
POSITIVE INDUCTIVE-RECURSIVE DEFINITIONS
}

\author{
NEIL GHANI, LORENZO MALATESTA, AND FREDRIK NORDVALL FORSBERG
}

University of Strathclyde, UK

e-mail address: ng@cis.strath.ac.uc, \{lorenzo.malatesta,fredrik.nordvall-forsberg\}@strath.ac.uk

\begin{abstract}
A new theory of data types which allows for the definition of types as initial algebras of certain functors $\operatorname{Fam}(\mathbb{C}) \rightarrow \operatorname{Fam}(\mathbb{C})$ is presented. This theory, which we call positive inductive-recursive definitions, is a generalisation of Dybjer and Setzer's theory of inductive-recursive definitions within which $\mathbb{C}$ had to be discrete - our work can therefore be seen as lifting this restriction. This is a substantial endeavour as we need to not only introduce a type of codes for such data types (as in Dybjer and Setzer's work), but also a type of morphisms between such codes (which was not needed in Dybjer and Setzer's development). We show how these codes are interpreted as functors on $\operatorname{Fam}(\mathbb{C})$ and how these morphisms of codes are interpreted as natural transformations between such functors. We then give an application of positive inductive-recursive definitions to the theory of nested data types and we give concrete examples of recursive functions defined on universes by using their elimination principle. Finally we justify the existence of positive inductiverecursive definitions by adapting Dybjer and Setzer's set-theoretic model to our setting.
\end{abstract}

\section{INTRODUCTION}

Inductive types are the bricks of dependently typed programming languages: they represent the building blocks on which any other type is built. The mortar the dependently typed programmer has at her disposal for computation with dependent types is recursion. Usually, a type $A$ is defined inductively, and then terms or types can be defined recursively over the structure of $A$. The theory of inductive-recursive definitions Dyb00, DS99] explores the simultaneous combination of these two basic ingredients, pushing the limits of the theoretical foundations of data types.

The key example of an inductive-recursive definition is Martin-Löf's universe à la Tarski [ML84]. A type $U$ consisting of codes for small types is introduced, together with a decoding function $T$, which maps codes to the types they denote. The definition is both inductive and recursive; the type $U$ is defined inductively, and the decoding function $T$ is

2012 ACM CCS: [Theory of computation]: Logic-Type theory; Semantics and reasoning-Program constructs - Type structure; Semantics and reasoning-Program semantics - Categorical semantics.

Key words and phrases: Martin-Löf Type theory, data types, induction-recursion, initial-algebra semantics.

This work was supported by the Engineering and Physical Sciences Research Council [grant numbers $\mathrm{EP} / \mathrm{G} 033056 / 1, \mathrm{EP} / \mathrm{K} 023837 / 1]$. 
defined recursively on the way the elements of $U$ are generated. The definition needs to be simultaneous, since the introduction rules for $U$ refer to $T$. We illustrate this by means of a concrete example: say we want to define a data type representing a universe containing a name for the natural numbers, closed under $\Sigma$-types. Such a universe will be the smallest family of sets $(U, T)$ satisfying the following equations

$$
\begin{array}{ll}
U & =1+\Sigma u: U \cdot T u \rightarrow U \\
T(\operatorname{inl} *) & =\mathbb{N} \\
T(\operatorname{inr}(u, f)) & =\Sigma x: T u \cdot T(f x)
\end{array}
$$

In this definition we see how ground types and the type constructor $\Sigma$ are reflected in $U$. The left summand of the right hand side of the equation defining $U$ is a code for the type of natural numbers, while the right summand is a code reflecting $\Sigma$-types. Indeed the name of a the type $\Sigma A B$ for $A$ : Set, $B: A \rightarrow$ Set in the universe $(U, T)$ will consists of a name in $U$ for the type $A$, i.e. an element $u: U$, and a function $f: T u \rightarrow U$ representing the $A$-indexed family of sets $B$. The decoding function $T$ maps elements of $U$ to types according to the description above: the code for natural numbers decodes to the set of natural numbers $\mathbb{N}$ while an element $(u, f)$ of the right summand decodes to the $\Sigma$-type it denotes. Other examples of inductive-recursive definitions have also appeared in the literature, such as e.g. Martin-Löf's computability predicates [ML72] or Aczel's Frege structures [Acz80]. Lately the use of inductive-recursive definitions to encode invariants in ordinary data structures has also been considered [EHA09].

Dybjer's Dyb00 insight was that these examples are instances of a general notion, which Dybjer and Setzer [DS99] later found a finite axiomatisation of. Their theory of inductive-recursive definitions IR consists of: (i) a representation of types as initial algebras of functors; and (ii) a grammar for defining such functors. Elements of the grammar are called IR codes, while functors associated to IR codes are called IR functors. The theory naturally covers simpler inductive types such as lists, trees, vectors, red-black trees etc. as well. Dybjer and Setzer [DS03 then gave an initial algebra semantics for IR codes by showing that IR functors are naturally defined on the category $\operatorname{Fam}(D)$ of families of elements of a (possibly large) type $D$ and that these functors do indeed have initial algebras. More generally, abstracting on the families construction and the underlying families fibration $\pi: \operatorname{Fam}(D) \rightarrow$ Set, we have recently shown how to interpret IR functors in an arbitrary fibration endowed with the appropriate structure [GMNFS13. In this article, we will only consider the families fibration.

There is, however, a complication. When interpreting IR functors such as those building universes closed under dependent products, the mixture of covariance and contravariance intrinsic in the $\Pi$ operator forces one to confine attention to functors Fam $|\mathbb{C}| \rightarrow \operatorname{Fam}|\mathbb{C}|$ or, equivalently, to work with only those morphisms between families which are commuting triangles. More abstractly, as we have shown GMNFS13, this corresponds to working in the split cartesian fragment of the families fibration $\pi: \operatorname{Fam}(\mathbb{C}) \rightarrow$ Set, i.e. to only consider those morphisms in Fam $(\mathbb{C})$ which represent strict reindexing. In this paper we remove this constraint and hence explore a further generalization of IR, orthogonal to the one proposed in Ghani et al. GMNFS13. We investigate the necessary changes of IR needed to provide a class of codes which can be interpreted as functors $\operatorname{Fam}(\mathbb{C}) \rightarrow \operatorname{Fam}(\mathbb{C})$. This leads us to consider a new variation $\mathrm{IR}^{+}$of inductive-recursive definitions which we call positive inductive-recursive definitions. The most substantial aspect of this new theory is that in 
order to define these new codes, one needs also to define the morphisms between codes. This is no handle-turning exercise!

We first recall Dybjer and Setzer's theory of inductive-recursive definitions (Section 2). To develop the theory we then introduce a syntax and semantics consisting of $\mathrm{IR}^{+}$codes and their morphisms, and an explanation how these codes are interpreted as functors $\operatorname{Fam}(\mathbb{C}) \rightarrow \operatorname{Fam}(\mathbb{C})$, where $\mathbb{C}$ is an arbitrary category (Section 3 ). We then illustrate the stronger elimination principles that are possible for positive inductive-recursive definitions. We consider several examples of catamorphisms that are not possible with ordinary inductive-recursive definitions (Section 4). As a practical application, we use positive inductive-recursive definitions to shed new light on nested data types (Section 5). We formally compare $\mathrm{IR}^{+}$with the existing theory of $\mathrm{IR}$ (Section 6), and adapt Dybjer and Setzer's model construction to our setting (Section 7). The material in this paper has been formalised in Agda GMNF14.

The paper uses a mixture of categorical and type theoretic constructions. However, the reader should bear in mind that the foundations of this paper are type theoretic. In other words, all constructions should be understood to take place in extensional Martin-Löf type theory with one universe Set. This is entirely standard in the literature. The one exception is the use of a Mahlo cardinal required to prove that positive inductive recursive functors have initial algebras in Section 7. It should be emphasised that the Mahlo cardinal is only used to justify the soundness of the theory, and does not play any computational role. We refer the interested reader to Dybjer and Setzer [DS99] - they use a Mahlo cardinal for the same purpose - for the technical details. We also use fibrational terminology occasionally when we feel it adds insight, however readers not familiar with fibrations can simply ignore such comments.

\section{INDUCTIVE-RECURSIVE DEFINITIONS}

In increasing complexity and sophistication, inductive definitions, indexed inductive definitions and inductive-recursive definitions encode more and more information about the data structures in question into the type itself. Being situated at the top of this hierarchy, inductive-recursive definitions provide a unifying theoretical framework for many different forms of data types. Indeed, both inductive and indexed inductive definitions are simple instances of IR $\mathrm{GHM}^{+} 13$.

The original presentation of induction-recursion given by Dybjer Dyb00 was as a schema. Dybjer and Setzer [DS99] further developed the theory to internalize the concept of an inductive-recursive definition. They developed a finite axiomatization of the theory through the introduction of a special type of codes for inductive-recursive definitions. The following axiomatization, which closely follows Dybjer and Setzer [DS99, presents the syntax of IR as an inductive definition.

Definition 2.1 (IR codes). Let $D$ be a (possibly large) type. The type of $\mathrm{IR}(D)$ codes has the following constructors:

$$
\begin{gathered}
\frac{d: D}{\iota d: \operatorname{IR}(D)} \\
\frac{A: \text { Set } f: A \rightarrow \operatorname{IR}(D)}{\sigma_{A} f: \operatorname{IR}(D)}
\end{gathered}
$$




$$
\frac{A: \text { Set } F:(A \rightarrow D) \rightarrow \operatorname{IR}(D)}{\delta_{A} F: \operatorname{IR}(D)}
$$

This is the syntax of induction-recursion - it is quite remarkable in our opinion that this most powerful of theories of data types can be presented in such a simple fashion. These rules have been written in natural-deduction style and we may use the ambient type theory to define, for example, the function $f$ in the code $\sigma_{A} f$. An example of an IR code is given in Example 2.5; this code represents the universe containing the natural numbers and closed under $\Sigma$-types given in Equation (1.1). We now turn to the semantics of inductionrecursion: we interpret IR codes as functors, and to this end, we use the standard families construction Fam from category theory. We start recalling the definition of the category $\operatorname{Fam}(\mathbb{C})$ of families of objects of a category $\mathbb{C}$.

Definition 2.2. Given a category $\mathbb{C}$, the category $\operatorname{Fam}(\mathbb{C})$ has objects pairs $(X, P)$ where $X$ is a set and $P: X \rightarrow \mathbb{C}$ is a functor which we can think of as an $X$-indexed family of objects of $\mathbb{C}$. A morphism from $(X, P)$ to $(Y, Q)$ is a pair $(h, k)$ where $h: X \rightarrow Y$ is a function, and $k: P \rightarrow Q \circ h$ is a natural transformation.

Of course, the naturality condition in the definition of a morphism of families is vacuous as the domains of the functors in question are discrete.

Remarks 2.3. For any category $\mathbb{C}$, the category $\operatorname{Fam}(\mathbb{C})$ always has rich structure:

- Fam $(\mathbb{C})$ is fibred over Set (see e.g. Jacobs [Jac99]). We omit the definitions here, but recall the standard splitting cleavage of the fibration $\pi: \operatorname{Fam}(\mathbb{C}) \rightarrow$ Set which is relevant later: a morphism $(h, k):(X, P) \rightarrow(Y, Q)$ is a split cartesian morphism if $k$ is a family of identity morphisms, i.e. if $P=Q \circ h$.

- $\operatorname{Fam}(\mathbb{C})$ is the free set indexed coproduct completion of $\mathbb{C}$; that is $\operatorname{Fam}(\mathbb{C})$ has all set indexed coproducts and there is an embedding $\mathbb{C} \rightarrow$ Fam $(\mathbb{C})$ universal among functors $F$ : $\mathbb{C} \rightarrow \mathbb{D}$ where $\mathbb{D}$ is a category with set indexed coproducts. Given an $A$-indexed collection of objects $\left(X_{a}, P_{a}\right)_{a: A}$ in $\operatorname{Fam}(\mathbb{C})$, its $A$-indexed coproduct is the family $\sum_{a: A}\left(X_{a}, P_{a}\right)=$ $\left(\sum_{a: A} X_{a},\left[P_{a}\right]_{a: A}\right)$.

- $\operatorname{Fam}(\mathbb{C})$ is cocomplete if and only if $\mathbb{C}$ has all small connected colimits (Carboni and Johnstone [CJ95, dual of Prop. 2.1]).

- Fam is a functor CAT $\rightarrow$ CAT; given $F: \mathbb{C} \rightarrow \mathbb{D}$, we get a functor $\operatorname{Fam}(F): \operatorname{Fam}(\mathbb{C}) \rightarrow$ $\operatorname{Fam}(\mathbb{D})$ by composition: $\operatorname{Fam}(F)(X, P)=(X, F \circ P)$. Here CAT is the category of large categories.

When $\mathbb{C}$ is a discrete category, a morphism between families $(X, P)$ and $(Y, Q)$ in $\operatorname{Fam}(\mathbb{C})$ consists of a function $h: X \rightarrow Y$ such that $P x=Q(h x)$ for all $x$ in $X$. From a fibrational perspective, this amounts to the restriction to the split cartesian fragment Fam $|\mathbb{C}|$ of the fibration $\pi: \operatorname{Fam}(\mathbb{C}) \rightarrow$ Set, for $\mathbb{C}$ an arbitrary category. This observation is crucial for the interpretation of IR codes as functors. Indeed, given a type $D$, which we think of as the discrete category $|D|$ (with objects terms of type $D$ ), we interpret IR codes as functors $\operatorname{Fam}|D| \rightarrow \operatorname{Fam}|D|$.

Theorem 2.4 (IR functors [DS03]). Let $D$ be a (possibly large) type. Every code $\gamma: \operatorname{IR}(D)$ induces a functor

$$
\llbracket \gamma \rrbracket: \text { Fam }|D| \rightarrow \text { Fam }|D|
$$


Proof. We define $\llbracket \gamma \rrbracket:$ Fam $|D| \rightarrow$ Fam $|D|$ by induction on the structure of the code $\gamma$. We first give the action on objects:

$$
\begin{aligned}
\llbracket \iota c \rrbracket(X, P) & =\left(1, \lambda_{-} . c\right) \\
\llbracket \sigma_{A} f \rrbracket(X, P) & =\sum_{a: A} \llbracket f a \rrbracket(X, P) \\
\llbracket \delta_{A} F \rrbracket(X, P) & =\sum_{g: A \rightarrow X} \llbracket F(P \circ g) \rrbracket(X, P)
\end{aligned}
$$

We now give the action on morphisms. Let $(h$, id $):(X, P) \rightarrow(Y, Q)$ be a morphism in Fam $|D|$, i.e. $h: X \rightarrow Y$ and $Q \circ h=P$.

$$
\begin{aligned}
\llbracket \iota c \rrbracket(h, \text { id }) & =\left(\mathrm{id}_{1}, \text { id }\right) \\
\llbracket \sigma_{A} f \rrbracket(h, \mathrm{id}) & =\left[\mathrm{in}_{a} \circ \llbracket f a \rrbracket(h, \mathrm{id})\right]_{a: A} \\
\llbracket \delta_{A} F \rrbracket(h, \mathrm{id}) & =\left[\mathrm{in}_{h \circ g} \circ \llbracket F(Q \circ h \circ g) \rrbracket(h, \text { id })\right]_{g: A \rightarrow X}
\end{aligned}
$$

Here, the last line type checks $Q \circ h=P$ since $D$ is discrete. Hence

$$
Q \circ h \circ g=P \circ g
$$

and we can apply the induction hypothesis.

Note how the interpretation of both $\sigma$ and $\delta$ codes makes essential use of coproducts of families as defined in Remarks 2.3. In particular, the interpretation of a code $\delta_{A} F$ uses as the coproduct's index set the function space $A \rightarrow X$, which is a set since both $A$ and $X$ are.

Ghani et al. [GHM $\left.{ }^{+} 13\right]$ introduces morphisms between (small) IR codes. The morphisms are chosen to make the interpretation function $\llbracket-\rrbracket: \operatorname{IR}(D) \rightarrow($ Fam $|D| \rightarrow$ Fam $|D|)$ full and faithful. Thus, transporting composition and identity along this function makes $\operatorname{IR}(D)$ into a category, and $\llbracket-\rrbracket: \operatorname{IR}(D) \rightarrow(\operatorname{Fam}|D| \rightarrow$ Fam $|D|)$ can really be seen as a full and faithful functor. We will draw inspiration from this in Section 3 when we generalise the semantics to endofunctors on $\operatorname{Fam}(\mathbb{C})$ for possibly non-discrete categories $\mathbb{C}$. Note however that the definition of morphisms between codes we give here differs from the one appearing in Ghani et al. $\left.\mathrm{GHM}^{+} 13\right]$. The key idea of the latter is a characterization of the interpretation of $\delta$ codes as left Kan extensions. In our more general setting where $\mathbb{C}$ can be a non-discrete category, this characterization fails. As a consequence, we lose the full and faithfulness of the interpretation functor $\llbracket-\rrbracket$ and we have to prove by hand that the set of codes and morphisms between them actually is a category. Full and faithfulness of the interpretation is convenient and desirable, and often simplifies calculations. Nonetheless, it is not an essential property, and we manage to make do without it.

We call a data type inductive-recursive if it is the initial algebra of a functor induced from an IR code. Let us look at some examples.

Example 2.5 (A universe closed under dependent sums). In the introduction, we introduced a universe in Equation (1.1), containing the natural numbers and closed under $\Sigma$ types, and claimed that this universe can be defined via an inductive-recursive definition. Indeed, one can easily write down a code $\gamma_{\mathbb{N}, \Sigma}: \operatorname{IR}($ Set $)$ for a functor that will have such a universe as its initial algebra:

$$
\gamma_{\mathbb{N}, \Sigma}:=\iota \mathbb{N}+{ }_{\mathrm{IR}} \delta_{1}\left(X \mapsto \delta_{X *}(Y \mapsto \iota \Sigma(X *) Y)\right): \operatorname{IR}(\text { Set })
$$

Here we have used $\gamma+\mathrm{IR} \gamma^{\prime}:=\sigma_{2}\left(0 \mapsto \gamma ; 1 \mapsto \gamma^{\prime}\right)$ to encode a binary coproduct as a 2indexed coproduct. Also, in the above, note that $X: 1 \rightarrow$ Set and so $X *$ is simply the 
application of $X$ to the canonical element of 1 . If we decode $\gamma_{\mathbb{N}, \Sigma}$, we get a functor which satisfies

$$
\llbracket \gamma_{\mathbb{N}, \Sigma} \rrbracket(U, T) \cong\left(1+\Sigma u: U . T(u) \rightarrow U, \operatorname{inl}_{-} \mapsto \mathbb{N} ; \operatorname{inr}(u, f) \mapsto \Sigma x: T(u) \cdot T(f(x))\right)
$$

so that the initial algebra $(U, T)$ of $\llbracket \gamma_{\mathbb{N}, \Sigma} \rrbracket$, which satisfies $(U, T) \cong \llbracket \gamma_{\mathbb{N}, \Sigma} \rrbracket(U, T)$ by Lambek's Lemma, indeed satisfies Equation (1.1).

Example 2.6 (A universe closed under dependent function spaces). In the same way, we can easily write a down a code for a universe closed under П-types:

$$
\gamma_{\mathbb{N}, \Pi}:=\iota \mathbb{N}+_{\mathrm{IR}} \delta_{1}\left(X \mapsto \delta_{X *}(Y \mapsto \iota \Pi(X *) Y)\right): \operatorname{IR}(\text { Set })
$$

Even though this looks extremely similar to the code in the previous example, we will see in the next section that there is a big semantic difference between them.

\section{Positive Inductive-Recursive Definitions}

Theorem 2.4 tells us that IR codes can be interpreted as functors on families built over a discrete category. What happens if we try to interpret IR codes on the category $\operatorname{Fam}(\mathbb{C})$, and not just on the subcategory Fam $|\mathbb{C}|$, whose morphisms are the split cartesian ones only? Consider the following morphism in Fam $|\mathbb{C}|$ :

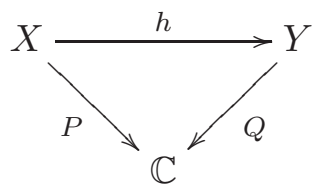

What if the diagram above does not commute on the nose, since $\mathbb{C}$ is not simply a discrete category, but a category whose intrinsic structure we want to keep track of? For instance, it is natural to require that the diagram above only commutes up to isomorphism, i.e. $P(x) \cong$ $Q(h(x))$ instead of $P(x)=Q(h(x))$. What structure is required to interpret inductiverecursive definitions in this larger category? The problem is that if we allow for more general morphisms, we can not prove functoriality of the semantics of a $\delta$ code as it stands anymore: it is essential to have an actual equality on the second component of a morphism in $\operatorname{Fam}(\mathbb{C})$ in order to have a sound semantics.

In this section we propose a new axiomatization which enables us to solve this problem. This new theory, which we dub positive inductive-recursive definitions, abbreviated $\mathrm{IR}^{+}$, represents a generalization of $I R$ which allows the interpretation of codes as functors defined on $\operatorname{Fam}(\mathbb{C})$ for an arbitrary category $\mathbb{C}$. In particular, if we choose $\mathbb{C}$ to be a groupoid, i.e. a category where every morphism is an isomorphism, we get triangles commuting up to isomorphism as morphisms in $\operatorname{Fam}(\mathbb{C})$.

3.1. Syntax and Semantics of $\mathbb{R}^{+}(\mathbb{C})$. The crucial insight which guides us when introducing the syntax of $\mathrm{IR}^{+}$is to deploy proper functors in the introduction rule of a $\delta$ code. This enables us to remove the restriction on morphisms within inductive recursive definitions; indeed, if we know that $F:(A \rightarrow \mathbb{C}) \rightarrow \mathbb{R}^{+}(\mathbb{C})$ is a functor, and not just a function, we do not have to rely on an identity in Equation (2.1), but we can use the second component of a morphism $(h, k):(X, P) \rightarrow(Y, Q)$ in $\operatorname{Fam}(\mathbb{C})$ to get a map $P \circ g \rightarrow Q \circ h \circ g$; then we can use the fact that $F$ is a functor to get a morphism between codes $F(P \circ g) \rightarrow F(Q \circ h \circ g)$. 
But, now we have to roll up our sleeves. For $F:(A \rightarrow \mathbb{C}) \rightarrow \mathbb{I}^{+}(\mathbb{C})$ to be a functor, we need both $A \rightarrow \mathbb{C}$ and $\mathrm{IR}^{+}(\mathbb{C})$ to be categories. While it is clear how to make $A \rightarrow \mathbb{C}$ a category, turning $\mathrm{IR}^{+}(\mathbb{C})$ into a category entails defining both codes and morphisms between codes simultaneously, in an inductive-inductive fashion NFS12, NF13. We give an axiomatic presentation of $\mathrm{IR}^{+}$analogously to the one given in Section 2 for the syntax of IR; however we now have mutual introduction rules to build both the type of $\mathrm{IR}^{+}(\mathbb{C})$ codes and the type of $\mathrm{IR}^{+}(\mathbb{C})$ morphisms, for $\mathbb{C}$ a given category. The semantics we give then explains how $\mathbb{R}^{+}(\mathbb{C})$ codes can be interpreted as functors on $\operatorname{Fam}(\mathbb{C})$, while $\mathrm{IR}^{+}(\mathbb{C})$ morphisms between such codes can be interpreted as natural transformations.

Definition 3.1. Given a category $\mathbb{C}$ we simultaneously define the type $\mathbb{R}^{+}(\mathbb{C})$ of positive inductive-recursive codes on $\mathbb{C}$, and the type of morphisms between these codes $\operatorname{Hom}_{\mathbb{R}^{+}(\mathbb{C})}\left(-,{ }_{-}\right): \mathrm{IR}^{+}(\mathbb{C}) \rightarrow \mathrm{IR}^{+}(\mathbb{C}) \rightarrow$ type as follows:

- $\mathrm{IR}^{+}(\mathbb{C})$ codes:

$$
\begin{gathered}
\frac{c: \mathbb{C}}{\iota c: I \mathrm{R}^{+}(\mathbb{C})} \\
\frac{A: \text { Set } \quad f: A \rightarrow \mathrm{I}^{+}(\mathbb{C})}{\sigma_{A} f: \mathrm{IR}^{+}(\mathbb{C})} \\
\frac{A: \text { Set } \quad F:(A \rightarrow \mathbb{C}) \rightarrow \mathrm{IR}^{+}(\mathbb{C})}{\delta_{A} F: \mathrm{IR}^{+}(\mathbb{C})}
\end{gathered}
$$

- $\mathrm{IR}^{+}(\mathbb{C})$ morphisms:$$
\frac{f: \operatorname{Hom}_{\mathbb{C}}\left(c, c^{\prime}\right)}{(\iota \Rightarrow \iota)(f): \operatorname{Hom}_{\mathbb{R}^{+}(\mathbb{C})}\left(\iota c, \iota c^{\prime}\right)}
$$$$
\frac{\alpha: A \rightarrow B \quad \rho: \prod_{x: A} \operatorname{Hom}_{\mathbb{R}^{+}(\mathbb{C})}(f(x), g(\alpha(x)))}{(\sigma \Rightarrow \sigma)(\alpha, \rho): \operatorname{Hom}_{\mathbb{R}^{+}(\mathbb{C})}\left(\sigma_{A} f, \sigma_{B} g\right)}
$$$$
\frac{\alpha: B \rightarrow A \quad \rho: \operatorname{Nat}(F, G(-\circ \alpha))}{(\delta \Rightarrow \delta)(\alpha, \rho): \operatorname{Hom}_{\mathbb{R}^{+}(\mathbb{C})}\left(\delta_{A} F, \delta_{B} G\right)}
$$

In the last clause, we have indicated with $\operatorname{Nat}(F, G(-\circ \alpha))$ the collection of natural transformations between the functors $F$ and $G(-\circ \alpha):(A \rightarrow \mathbb{C}) \rightarrow \mathbb{I}^{+}(\mathbb{C})$. Note also the contravariant twist in the type of $\alpha: B \rightarrow A$ in this clause.

We need to make sure that Definition 3.1 really defines a category, i.e. that composition of $\mathrm{IR}^{+}$morphisms can be defined, and that it is associative and has identities. This can be proved by recursion on the structure of morphisms:

Lemma 3.2. Let $\mathbb{C}$ be a category. Then $\mathrm{IR}^{+}(\mathbb{C})$ is a category with morphisms given by $\operatorname{Hom}_{\mathrm{IR}^{+}(\mathbb{C})}$.

Proof. We define $\mathrm{id}_{x}^{+}: \operatorname{Hom}_{\mathrm{R}^{+}(\mathbb{C})}(x, x)$ by recursion on $x$ :

$$
\begin{aligned}
\mathrm{id}_{\iota c}^{+} & =(\iota \Rightarrow \iota)\left(\mathrm{id}_{c}\right) \\
\mathrm{id}_{\sigma_{A} f}^{+} & =(\sigma \Rightarrow \sigma)\left(\mathrm{id}_{A}, \lambda a \cdot \mathrm{id}_{f(a)}^{+}\right) \\
\mathrm{id}_{\delta_{A} F}^{+} & =(\delta \Rightarrow \delta)\left(\mathrm{id}_{A}, \lambda h \cdot \mathrm{id}_{F(h)}^{+}\right)
\end{aligned}
$$


Composition $-{ }_{\mathrm{IR}^{+}}$- $: \operatorname{Hom}_{\mathrm{R}^{+}(\mathbb{C})}(y, z) \rightarrow \operatorname{Hom}_{\mathrm{IR}^{+}(\mathbb{C})}(x, y) \rightarrow \operatorname{Hom}_{\mathrm{IR}^{+}(\mathbb{C})}(x, z)$ is defined by recursion on $f: \operatorname{Hom}_{\mathbb{I R}^{+}(\mathbb{C})}(y, z)$ and $g: \operatorname{Hom}_{\mathbb{R}^{+}(\mathbb{C})}(x, y)$ :

$$
\begin{aligned}
(\iota \Rightarrow \iota)(f) \circ_{\mathbb{I}^{+}}(\iota \Rightarrow \iota)(g) & =(\iota \Rightarrow \iota)\left(f \circ \circ_{\mathbb{C}} g\right) \\
(\sigma \Rightarrow \sigma)(\alpha, \rho) \circ_{\mathbb{R}^{+}}(\sigma \Rightarrow \sigma)(\beta, \tau) & =(\sigma \Rightarrow \sigma)\left(\alpha \circ \beta, \lambda x \cdot \rho(\alpha(x)) \circ_{\mathbb{R}^{+}} \tau(x)\right) \\
(\delta \Rightarrow \delta)(\alpha, \rho) \circ_{\mathbb{R}^{+}}(\delta \Rightarrow \delta)(\beta, \tau) & =(\delta \Rightarrow \delta)\left(\beta \circ \alpha, \lambda h . \rho(h \circ \beta) \circ_{\mathbb{R}^{+}} \tau(h)\right)
\end{aligned}
$$

Three more straightforward inductions prove that composition is associative, and that $\mathrm{id}^{+}$ is both a left and a right unit for composition.

We now explain how each code $\gamma: \mathbb{I R}^{+}(\mathbb{C})$ is interpreted as an endofunctor

$$
\llbracket \gamma \rrbracket: \operatorname{Fam}(\mathbb{C}) \rightarrow \operatorname{Fam}(\mathbb{C})
$$

We call a functor which is isomorphic to a functor induced by an $\mathrm{IR}^{+}$code an $\mathrm{IR}^{+}$functor. The semantics of $\mathrm{IR}^{+}$closely follows the one given in Section 2; as before we make essential use of coproducts in $\operatorname{Fam}(\mathbb{C})$. Having said that, the crucial feature which separates the semantics of $\mathrm{IR}^{+}$from the semantics of IR is the following: when explaining the semantics of IR, we first interpret IR codes as functors and then later define morphisms between codes. We can then interpret the morphisms as natural transformations between the corresponding functors. In $\mathrm{IR}^{+}$, the type of codes and the type of morphisms between codes are simultaneously defined in an inductive-inductive way, and therefore they are also decoded simultaneously as functors and natural transformations respectively. This is exactly what the elimination principle for an inductive-inductive definition gives.

In the following theorem, note that there is no restriction on the category $\mathbb{C}$ - all structure that we need comes for free from the families construction Fam.

Theorem 3.3 ( $\mathrm{IR}^{+}$functors). Let $\mathbb{C}$ be an arbitrary category.

(i) Every code $\gamma: \mathbb{R}^{+}(\mathbb{C})$ induces a functor $\llbracket \gamma \rrbracket: \operatorname{Fam}(\mathbb{C}) \rightarrow \operatorname{Fam}(\mathbb{C})$.

(ii) Every morphism $\rho: \mathbb{I R}^{+}(\mathbb{C})\left(\gamma, \gamma^{\prime}\right)$ for codes $\gamma, \gamma^{\prime}: \mathbb{I}^{+}(\mathbb{C})$ gives rise to a natural transformation $\llbracket \rho \rrbracket: \llbracket \gamma \rrbracket \stackrel{\cdot}{\longrightarrow} \llbracket \gamma^{\prime} \rrbracket$.

Proof. While the action on objects is the same for both $\mathrm{IR}^{+}$and IR functors, the action on morphisms is different when interpreting a code of type $\delta_{A} F$ : in the semantics of $\mathrm{IR}^{+}$ we exploit the fact that $F:(A \rightarrow \mathbb{C}) \rightarrow \mathrm{IR}^{+}(\mathbb{C})$ is now a functor, so that it also has an action on morphisms (which we, for the sake of clarity, write $F_{\rightarrow}$ ). We give the action of $\mathrm{IR}^{+}$functors on morphisms only, and refer to the semantics given in Theorem 2.4 for the action on objects of $\operatorname{Fam}(\mathbb{C})$.

The action on morphisms is given as follows. Let $(h, k):(X, P) \rightarrow(Y, Q)$ in $\operatorname{Fam}(\mathbb{C})$. We define $\llbracket \gamma \rrbracket(h, k): \llbracket \gamma \rrbracket(X, P) \rightarrow \llbracket \gamma \rrbracket(Y, Q)$ by recursion on $\gamma$ :

$$
\begin{aligned}
\llbracket \iota c \rrbracket(h, k) & =\left(\mathrm{id}_{1}, \mathrm{id}_{c}\right) \\
\llbracket \sigma_{A} f \rrbracket(h, k) & =\left[\mathrm{in}_{a} \circ \llbracket f a \rrbracket(h, k)\right]_{a: A} \\
\llbracket \delta_{A} F \rrbracket(h, k) & =\left[\operatorname{in}_{h \circ g} \circ \llbracket F(Q \circ h \circ g) \rrbracket(h, k) \circ \llbracket F_{\rightarrow}\left(g^{*}(k)\right) \rrbracket_{(X, P)}\right]_{g: A \rightarrow X}
\end{aligned}
$$

In the last clause $g^{*}(k): P \circ g \longrightarrow Q \circ h \circ g$ is the natural transformation with component $g^{*}(k)_{a}=k_{g a}: P(g a) \rightarrow Q(k(g a))$; note that such a natural transformation is nothing but the vertical morphism above $A$ obtained by reindexing ( $\left.\mathrm{id}_{X}, k\right)$ along $g$ in the families fibration $\pi: \operatorname{Fam}(\mathbb{C}) \rightarrow$ Set. 
We now explain how an $\mathrm{IR}^{+}$morphism $\rho: \gamma \rightarrow \gamma^{\prime}$ is interpreted as a natural transformation $\llbracket \rho \rrbracket: \llbracket \gamma \rrbracket \rightarrow \llbracket \llbracket \gamma^{\prime} \rrbracket$ between $\mathrm{IR}^{+}$functors by specifying the component $\llbracket \rho \rrbracket_{(X, P)}$ at $(X, P)$ : Fam $(\mathbb{C})$. Naturality of these transformations can be proved by a routine diagram chase.

$$
\begin{aligned}
\llbracket(\iota \Rightarrow \iota)(f) \rrbracket_{(X, P)} & =\left(\mathrm{id}_{1}, f\right) \\
\llbracket(\sigma \Rightarrow \sigma)(\alpha, \rho) \rrbracket_{(X, P)} & =\left[\mathrm{in}_{\alpha(x)} \circ \llbracket \rho(x) \rrbracket_{(X, P)}\right]_{x: A} \\
\llbracket(\delta \Rightarrow \delta)(\alpha, \rho) \rrbracket_{(X, P)} & =\left[\operatorname{in}_{g \circ \alpha} \circ \llbracket \rho_{(P \circ g)} \rrbracket_{(X, P)}\right]_{g: A \rightarrow X}
\end{aligned}
$$

Remark 3.4. In the conference version of this paper GMNF13, we considered a different collection of morphisms; since more morphisms makes it easier to define codes, we tried to include as many morphisms as possible. As a result, the proof that $\operatorname{IR}^{+}(\mathbb{C})$ is a category becomes quite long and tedious, although straightforward. In this presentation, we have instead decided to restrict ourselves to the smallest possible "usable" combination of morphisms. It should be noted that our results are completely parametric in the choice of morphisms used; any collection that represents natural transformations between the codes works, as long as the identity morphisms and composition can be defined. The range spans all the way from no non-identity morphisms at all (in which case it is rather hard to define a functor $(A \rightarrow \mathbb{C}) \rightarrow \mathbb{I}^{+}(\mathbb{C})$ !) to taking $\operatorname{Hom}_{\mathbb{R}^{+}}(x, y)=\llbracket x \rrbracket \rightarrow \llbracket y \rrbracket$, which gives rise to a full and faithful interpretation by definition. The latter would mean that the interpretation $\llbracket-\rrbracket$ would need to be defined simultaneously with the codes, with the effect that the very definition of positive inductive-recursive definitions itself would be inductive-recursive. To avoid this stronger assumption in the metatheory, we prefer the current formulation, where the meta-theory only uses inductive-inductive definitions - a much weaker principle.

Let us now return to the examples from the end of Section 2 .

Example 3.5 (A universe closed under dependent sums in Fam(Set $\left.{ }^{\mathrm{op}}\right)$ ). In Example 2.5, we defined an ordinary IR code $\gamma_{\mathbb{N}, \Sigma}: \operatorname{IR}($ Set) for a universe closed under sigma types. We can extend this code to an $\mathrm{IR}^{+}$code

$$
\gamma_{\mathbb{N}, \Sigma}=\iota \mathbb{N}+{ }_{\mathrm{IR}} \delta_{1}\left(X \mapsto \delta_{X *}(Y \mapsto \iota \Sigma(X *) Y)\right): \mathrm{IR}^{+}\left(\mathrm{Set}^{\mathrm{op}}\right)
$$

where now $G:=Y \mapsto \iota \Sigma(X *) Y$ and $F:=X \mapsto \delta_{X *} G$ need to be functors. Given $f: Y \rightarrow Y^{\prime}$ in $X \rightarrow$ Set $^{\text {op }}$, i.e. an $X$-indexed collection of morphisms $f_{x}: Y(x) \rightarrow Y^{\prime}(x)$ in Set ${ }^{\mathrm{op}}$, we have $\Sigma x:(X *) \cdot f_{x}: \Sigma(X *) Y \rightarrow \Sigma(X *) Y^{\prime}$ in Set ${ }^{\mathrm{op}}$ so that we can define

$$
G(f): \iota \Sigma(X *) Y \rightarrow \iota \Sigma(X *) Y^{\prime}
$$

by $G(f)=(\iota \Rightarrow \iota)\left(\Sigma x:(X *) \cdot f_{x}\right)$.

We also need $F$ to be a functor. Given $f: X \rightarrow X^{\prime}$ in $1 \rightarrow$ Set $^{\text {op }}$, we need to define $F(f): \delta_{X *} G \rightarrow \delta_{X^{\prime} *} G$. According to Definition 3.1, it is enough to give an $\alpha: X^{\prime} * \rightarrow X *$ and a natural transformation $\rho$ from $G$ to $G(-\circ \alpha)$. We can choose $\alpha=f_{*}$, and $\rho$ to be the natural transformation whose component at $Y: X * \rightarrow$ Set $^{\text {op }}$ is given by $\rho_{Y}=(\iota \Rightarrow$ $\iota)\left(\left[\operatorname{in}_{f_{*} x}\right]_{x: X^{\prime} *}\right)$, where $\left[\operatorname{in}_{f_{*} x}\right]_{x: X^{\prime} *}: \Sigma\left(X^{\prime} *\right) Y \circ f_{*} \rightarrow \Sigma(X *) Y$. Notice that working in Set ${ }^{\text {op }}$ made sure that $f_{*}$ was going in the right direction.

Example 3.6 (A universe closed under dependent function spaces in Fam $\left(\mathrm{Set}^{\cong}\right)$ ). In Example 2.6, we saw how we could use induction-recursion to define a universe closed under $\Pi$-types in Fam $\mid$ Set|, using the following code:

$$
\gamma_{\mathbb{N}, \Pi}=\iota \mathbb{N}+_{\mathrm{IR}} \delta_{1}\left(X \mapsto \delta_{X *}(Y \mapsto \iota \Pi(X *) Y)\right): \operatorname{IR}(\text { Set })
$$


If we try to extend this to an $\mathrm{IR}^{+}$code in $\mathrm{Fam}(\mathrm{Set})$ or Fam(Set $\left.{ }^{\mathrm{op}}\right)$, we run into problems. Basically, given a morphism $f: X^{\prime} \rightarrow X$, we need to construct a morphism $\Pi X^{\prime}(Y \circ f) \rightarrow$ $\Pi X Y$, which of course is impossible if e.g. $X^{\prime}=0, X=1$, and $Y *=0$.

Hence the inherent contravariance in the $\Pi$-type means that $\gamma_{\mathbb{N}, \Pi}$ does not extend to a $\mathrm{IR}^{+}$(Set) or $\mathrm{IR}^{+}\left(\right.$Set $\left.^{\mathrm{op}}\right)$ code. However, if we move to the groupoid Set $\cong$, which is the subcategory of Set with only isomorphisms as morphisms, we do get an $\mathrm{IR}^{+}(\mathrm{Set} \stackrel{\cong}{\cong})$ code describing the universe in question, which is still living in a category beyond the strict category Fam $\mid$ Set $\mid$. It would be interesting to understand the relevance of positive induction-recursion to Homotopy Type Theory Uni13. where groupoids and their higher order relatives play such a prominent role.

\section{Stronger ELimination PRINCIPLES}

From Example 3.5 we know that the IR code $\gamma_{\mathbb{N}, \Sigma}$ defining a universe containing the set of natural numbers $\mathbb{N}$ and closed under $\Sigma$-type can be extended to a $\mathrm{IR}^{+}$code of type $\mathrm{IR}^{+}(\mathrm{Set} \cong$ $\cong$ or $\mathrm{IR}^{+}\left(\mathrm{Set}^{\mathrm{OP}}\right)$. Thus, the code $\gamma_{\mathbb{N}, \Sigma}$ can be interpreted as an endofunctor on $\mathrm{Fam}\left(\mathrm{Set}{ }^{\cong}\right)$ or on Fam(Set ${ }^{\text {op }}$ ) respectively. In this section we aim to explore by means of an example what the elimination principle for $\mathrm{IR}^{+}$codes can be used for: we show how the simple elaboration of the code $\gamma_{\mathbb{N}, \Sigma}$ to a code of type $\operatorname{IR}^{+}$(Set $\cong$ ) offers us the possibility to implement a more sophisticated recursion principle on the universe we are currently building.

Recall that from the perspective of initial algebra semantics, the elimination principle for a type is captured by the universal property of the initial algebra: if $F$ is an endofunctor and $\left(\mu_{F}, i n_{F}\right)$ its initial algebra, then we know that for any other algebra $(X, f)$ there exists a (unique) $F$-algebra homomorphism $\alpha_{f}: \mu_{F} \rightarrow X$ which makes the following diagram commute:

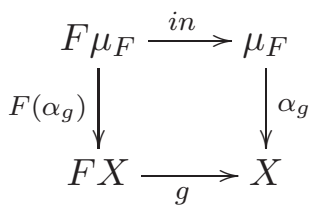

The initial property of $\left(\mu_{F}, i n_{F}\right)$ thus gives us a definition by recursion into any other type possessing the right $F$-algebra structure. By working in $\operatorname{Fam}(\mathbb{C})$ instead of $\operatorname{Fam}|\mathbb{C}|$, we are allowing many more algebras compared to ordinary inductive-recursive definitions, or put differently, we get a stronger elimination principle.

Example 4.1. To see why a stronger elimination principle is sometimes necessary, consider the initial algebra $\left(\left(U^{*}, T^{*}\right),\left(\mathrm{in}_{0}, \mathrm{in}_{1}\right)\right)$ for a code $\gamma_{1, \mathbb{N}, \Sigma}: \mathrm{IR}^{+}($Set $\stackrel{\cong}{\cong})$ representing a universe containing a set 1 with only one element, the set $\mathbb{N}$ of natural numbers and moreover closed under $\Sigma$-types. The universe $U^{*}$ contains many codes for "the same" set, up to isomorphism. For instance, it contains codes for each of the following isomorphic sets:

$$
\begin{aligned}
& 1 \cong(\Sigma 1) 1 \cong(\Sigma 1)(\Sigma 1) 1 \ldots \\
& \mathbb{N} \cong(\Sigma \mathbb{N}) 1 \cong(\Sigma 1) \mathbb{N} \cong(\Sigma 1)(\Sigma 1) \mathbb{N} \ldots
\end{aligned}
$$

Moreover, for each $\Sigma$-type the following isomorphism holds:

$$
(\Sigma z:(\Sigma x: A) B(x)) C(z) \cong(\Sigma x: A)(\Sigma y: B(a)) C(\langle x, y\rangle)
$$

Therefore, for each $\Sigma$ set with at least two nested $\Sigma$ 's, $U^{*}$ contains a code for both these ways to parenthesize a $\Sigma$-type. It might be advantageous to instead keep a single representative 
for each isomorphism class. We might hope to do so using the initiality of $\left(U^{*}, T^{*}\right)$, and indeed, the elimination principle for positive inductive-recursive definitions allows us to do exactly that.

First of all we need to decide what normal forms for elements in the universe we want. We can specify this by defining a predicate NF : $U^{*} \rightarrow$ Set on the universe $\left(U^{*}, T^{*}\right)$, which decides if a set is in normal form: we decree that the codes for the sets 1 and $\mathbb{N}$ are in normal form, and a code for $\Sigma A B$ is in normal form if $A$ is in normal form, $B(a)$ is in normal form for each $a: A, A$ is not 1 , and finally it is of the form of the right hand side of (4.1). There is of course some room for different choices here. Formally, and employing some cleverness in how we set things up, we can define the predicate by the elimination principle for $U^{*}$ by the following clauses:

$$
\begin{aligned}
\operatorname{NF}(\hat{1}) & =\top \\
\operatorname{NF}(\hat{\mathbb{N}}) & =\top \\
\operatorname{NF}(\hat{\Sigma} \hat{1} b) & =\perp \\
\operatorname{NF}(\hat{\Sigma} \hat{\mathbb{N}} b) & =\forall n: \mathbb{N} \cdot \operatorname{NF}(b(n)) \\
\operatorname{NF}\left(\left(\hat{\Sigma}\left(\hat{\Sigma} a^{\prime} b^{\prime}\right) b\right)\right. & =\perp
\end{aligned}
$$

We now define a new family $\left(U_{\mathrm{NF}}, T_{\mathrm{NF}}\right)$, containing sets in normal forms only, by letting

$$
\begin{aligned}
U_{\mathrm{NF}} & :=\left(\Sigma u: U^{*}\right) \operatorname{NF}(u) \\
T_{\mathrm{NF}}(u, p) & :=T^{*}(u)
\end{aligned}
$$

We can also define a $\operatorname{Fam}\left(\operatorname{Set}^{\stackrel{\cong}{ }}\right)$ morphism $(\phi, \eta): \llbracket \gamma_{1, \mathbb{N}, \Sigma} \rrbracket\left(U_{\mathrm{NF}}, T_{\mathrm{NF}}\right) \rightarrow\left(U_{\mathrm{NF}}, T_{\mathrm{NF}}\right)$ which endows $\left(U_{\mathrm{NF}}, T_{\mathrm{NF}}\right)$ with an $\llbracket \gamma_{1, \mathbb{N}, \Sigma} \rrbracket$-algebra structure. For this, is it crucial that we are working in $\operatorname{Fam}\left(\mathrm{Set}^{\cong}\right)$ and not Fam $\mid$ Set $\mid$, since we can only expect that a $\Sigma$-type of normal forms is isomorphic to a normal form, not equal to one; i.e. if $A$ is in normal form, and $B(a)$ is in normal form for all $a: A$, then $\Sigma A B$ is not necessary normal (as e.g. $A=1$ shows), but we can always find a normal form isomorphic to $\Sigma A B$. The function $\phi$ maps $A$ and $B$ to this normal form, and $\eta$ is a proof that it is indeed isomorphic to $\Sigma A B$. We only give the definition of $\phi: \llbracket \gamma_{1, \mathbb{N}, \Sigma} \rrbracket_{0}\left(U_{\mathrm{NF}}, T_{\mathrm{NF}}\right) \rightarrow U_{\mathrm{NF}}$ here; the definition of $\eta$ follows the same pattern.

$$
\begin{aligned}
& \phi(\hat{1})=(\hat{1}, *) \\
& \phi(\hat{\mathbb{N}})=(\hat{\mathbb{N}}, *) \\
& \phi(\hat{\Sigma}(\hat{1}, p) b)=\left(\pi_{0} b(*), \pi_{1} b(*)\right) \\
& \phi(\hat{\Sigma}(\hat{\mathbb{N}}, p) b)=\left(\hat{\Sigma} \hat{\mathbb{N}}\left(\pi_{0} \circ b\right), n \mapsto \pi_{1}(b(n))\right) \\
& \phi\left(\hat{\Sigma}\left(\hat{\Sigma} \hat{\mathbb{N}} b^{\prime}, p\right) b\right)=\left(\hat{\Sigma} \hat{\mathbb{N}}\left(n \mapsto \pi_{0}(\phi(\ldots))\right),\left(n \mapsto \pi_{1}(\phi(\ldots))\right)\right) \\
& \text { where } \phi(\ldots)=\phi\left(\hat{\Sigma}\left(b^{\prime}(n), p(n)\right)(y \mapsto b(n, y))\right) \\
& \phi\left(\hat{\Sigma}\left(\hat{\Sigma}(\hat{\Sigma} a b) b^{\prime}, p\right) c\right) \quad \text { impossible case by the def. of NF; we have } p: \perp \\
& \phi\left(\hat{\Sigma}\left(\hat{\Sigma} \hat{1} b^{\prime}, p\right) c\right) \quad \text { impossible case by the def. of NF; we have } p: \perp
\end{aligned}
$$


By initiality of $\left(U^{*}, T^{*}\right)$ we get a morphism (nf, correct) making the following diagram commute:

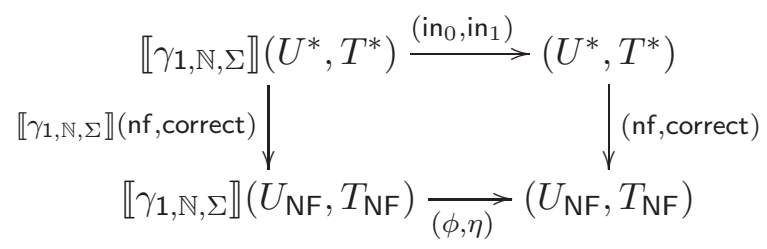

The map (nf, correct) recursively computes the normal form for each set in the universe $\left(U^{*}, T^{*}\right)$. Indeed, nf : $U^{*} \rightarrow U_{\mathrm{NF}}$ maps each name $u$ of a set $T(u)$ in the universe to the name of the corresponding set in normal form, while the natural transformation correct $_{u}$ : $T^{*}(u) \cong T_{\mathrm{NF}}(\operatorname{nf}(u))$ ensures that the code actually denotes isomorphic sets. Of course, we do not get (nf, correct) for free; defining $\phi$ and $\eta$ already amounts to most of the work for the full definition. The point is rather that initiality in $\operatorname{Fam}\left(\operatorname{Set}^{\cong}\right)$ is a definitional principle which allows us to define nf and correct. Furthermore, by using initiality, we can give a structured definition, where we only have to consider the separate cases in isolation.

Example 4.2. As another example of the use of elimination principles beyond ordinary inductive-recursive definitions, we can define functions between universes with different ground sets. Consider two universes $U_{1}, U_{2}$ closed under the same type-theoretic operations, but containing different ground sets $B_{1}, B_{2}$. Given a function $B_{1} \rightarrow B_{2}$, we would like to be able to extend this function to a function $U_{1} \rightarrow U_{2}$ between all of the two universes. For example, we could have a universe $\left(U_{\mathbb{N}, \Sigma}, T_{\mathbb{N}, \Sigma}\right)$, closed under $\Sigma$-types and containing the natural numbers $\mathbb{N}$, and another universe $\left(U_{\mathbb{Z}, \Sigma}, T_{\mathbb{Z}, \Sigma}\right)$ also closed under $\Sigma$-types but instead containing the integers $\mathbb{Z}$ as ground set. There ought to exist a function between them in Fam $\left(\mathrm{Set}^{\mathrm{oP}}\right.$ ) (the contravariance is needed for the negative occurrence of $U$ in the code for the sigma type), since clearly these two universes are closely related. By the elimination principle for positive inductive-recursive definitions, it suffices to provide a function between the ground sets, i.e. a function from $\mathbb{Z}$ into $\mathbb{N}$, for instance the absolute value function or the square function. In detail, every function $f: \mathbb{Z} \rightarrow \mathbb{N}$ induces a Fam(Set ${ }^{\text {op }}$-morphism

$$
\llbracket \gamma_{\mathbb{N}, \Sigma} \rrbracket\left(U_{\mathbb{Z}, \Sigma}, T_{\mathbb{Z}, \Sigma}\right) \longrightarrow\left(U_{\mathbb{Z}, \Sigma}, T_{\mathbb{Z}, \Sigma}\right)
$$

showing that $\left(U_{\mathbb{Z}, \Sigma}, T_{\mathbb{Z}, \Sigma}\right)$ has an $\llbracket \gamma_{\mathbb{N}, \Sigma} \rrbracket$-algebra structure. Therefore, initiality of $\left(U_{\mathbb{N}, \Sigma}, T_{\mathbb{N}, \Sigma}\right)$ gives us a map $\left(U_{\mathbb{N}, \Sigma}, T_{\mathbb{N}, \Sigma}\right) \rightarrow\left(U_{\mathbb{Z}, \Sigma}, T_{\mathbb{Z}, \Sigma}\right)$ which uses $f$ to recursively compute the embedding of $\left(U_{\mathbb{N}, \Sigma}, T_{\mathbb{N}, \Sigma}\right)$ into $\left(U_{\mathbb{Z}, \Sigma}, T_{\mathbb{Z}, \Sigma}\right)$.

\section{Applichtion: A Concrete Representation of Nested Types}

Nested data types AMU05 have been used to implement a number of advanced data types in languages which support higher-kinded types, such as the widely-used functional programming language Haskell. Among these data types are those with constraints, such as perfect trees [Hin00]; types with variable binding, such as untyped $\lambda$-terms [FPT99]; cyclic data structures GHUV06]; and certain dependent types [MM04].

A canonical example of a nested data type is Lam : Set $\rightarrow$ Set defined in Haskell as follows:

data $\operatorname{Lam} a=\operatorname{Var} a|\operatorname{App}(\operatorname{Lam} a)(\operatorname{Lam} a)| \operatorname{Abs}(\operatorname{Lam}($ Maybe a)) 
The type Lam a is the type of untyped $\lambda$-terms over variables of type a up to $\alpha$-equivalence. Here, the constructor Abs models the bound variable in an abstraction of type Lam a by the Nothing constructor of type Maybe a, and any free variable $\mathrm{x}$ of type a in an abstraction of type Lam a by the term Just $\mathrm{x}$ of type Maybe a; The key observation about the type Lam a is that elements of the type Lam (Maybe a) are needed to build elements of Lam a so that, in effect, the entire family of types determined by Lam has to be constructed simultaneously. Thus, rather than defining a family of inductive types, the type constructor Lam defines a type-indexed inductive family of types. The kind of recursion captured by nested types is a special case of non-uniform recursion [Bla00].

On the other hand, ordinary non-nested data types such as List a or Tree a can be represented as containers AAG05, Abb03. Recall that a container $(S, P)$ is given by a set $S$ of shapes, together with a family $P: S \rightarrow$ Set of positions. Each container gives rise to a functor $\llbracket S, P \rrbracket_{\text {Cont }}:$ Set $\rightarrow$ Set defined by $\llbracket S, P \rrbracket$ Cont $(X)=\Sigma s: S . P(s) \rightarrow X$. Since also nested data types such as Lam have type Set $\rightarrow$ Set, it make sense to ask the following question: Are nested data types representable as containers? There would be benefits of a positive answer, since container technology could then be applied to nested data types. For instance, we could operate on nested types using container operations such as the derivative, and classify the natural transformations between them. Note in particular that the canonical recursion operator fold for nested data types is a natural transformation.

We give a positive answer to the above question using $\mathrm{IR}^{+}$. As far as we are aware, this is a new result. We sketch our overall development as follows:

(i) We define a grammar Nest for defining nested types and a decoding function (-) : Nest $\rightarrow$ (Set $\rightarrow$ Set $) \rightarrow$ (Set $\rightarrow$ Set $)$. The data types we are interested in arise as initial algebras $\mu(N)$ for elements $N$ of the grammar.

(ii) We show that $(N)$ restricts to an endofunctor $(N)$ Cont : Cont $\rightarrow$ Cont on the category Cont of containers.

(iii) Noting that Cont $=\operatorname{Fam}\left(\operatorname{Set}^{\mathrm{op}}\right)$, we use $\mathrm{IR}^{+}$to define $(N)$ Cont. Hence by the results of this paper, $(N)_{\text {Cont }}$ has an initial algebra $\mu(N)_{\text {cont }}$. We finish by arguing that $\mu(N)=\llbracket \mu(N)$ Cont $\rrbracket$ Cont and hence that, indeed, nested types are containers.

A Grammar for Nested Types. We now present a grammar for defining nested data types. Since our point is not to push the theory of nested data types, but rather to illustrate an application of positive induction-recursion, we keep the grammar simple. The grammar we use is

$$
\mathcal{F}=\mathrm{Id}|K C| \mathcal{F}+\mathcal{F}|\mathcal{F} \times \mathcal{F}| \mathcal{F} \circledast \mathcal{F}
$$

where $C$ is any container. The intention is that Id stands for the identity functor mapping a functor to itself, $K C$ stands for the constant functor mapping any functor to the interpretation of the container $C,+$ and $\times$ stand for the coproduct and product of functors respectively, and $\circledast$ for the pointwise composition of functors. These intentions are formalised by a semantics for the elements of our grammar given as follows

$$
\begin{array}{ll}
(-) & : \text { Nest } \rightarrow(\text { Set } \rightarrow \text { Set }) \rightarrow(\text { Set } \rightarrow \text { Set }) \\
(\text { IId }) F & =F \\
(K C) F & =\llbracket C \rrbracket \text { Cont } \\
\left(\mathcal{F}_{0}+\mathcal{F}_{1}\right) F & =\left(\mathcal{F}_{0}\right) F+\left(\mathcal{F}_{1}\right) F \\
\left(\mathcal{F}_{0} \times \mathcal{F}_{1}\right) F & =\left(\mathcal{F}_{0}\right) F \times\left(\mathcal{F}_{1}\right) F \\
\left(\mathcal{F}_{0} \circledast \mathcal{F}_{1} D F\right. & =\left(\mathcal{F}_{0}\right) F \circ\left(\mid \mathcal{F}_{1}\right) F
\end{array}
$$


For example, the functor

$$
L F X=X+(F X \times F X)+F(X+1)
$$

whose initial algebra is the type Lam, is of the form $\left(N_{L}\right)$ with

$$
N_{L}=K I_{C}+(\mathrm{Id} \times \mathrm{Id})+(\mathrm{Id} \circledast(K M))
$$

where $I_{C}=\left(1,,_{-} \mapsto 1\right)$ is the container with one shape and one position, representing the identity functor on Set, and $M=(2, x \mapsto$ if $x$ then 1 else 0$)$ is the container with two shapes, the first one with one position, and the second one with no position. $M$ represents the functor on Set mapping $X$ to $X+1$.

Nested Types as Functors on Containers. The next thing on our agenda is to show that every element $N$ of Nest has an interpretation as an operator on containers $(N)_{\text {Cont }}$ : Cont $\rightarrow$ Cont, such that $(N)$ Cont is the restriction of $(N)$ to the subcategory of functors that are extension of containers. This is done easily enough by recursion on $N$, noting that containers are closed under coproduct, product and composition:

Lemma 5.1 ([AAG05]). Let $(S, P)$ and $\left(S^{\prime}, P^{\prime}\right)$ be containers. Define

$$
\begin{aligned}
(S, P)+\left(S^{\prime}, P^{\prime}\right) & :=\left(S+S^{\prime},\left[P, P^{\prime}\right]\right) \\
(S, P) \times\left(S^{\prime}, P^{\prime}\right) & :=\left(S \times S^{\prime},\left(s, s^{\prime}\right) \mapsto P(s)+P^{\prime}\left(s^{\prime}\right)\right) \\
(S, P) \circ\left(S^{\prime}, P^{\prime}\right) & :=\left(\Sigma s: S .\left(P(s) \rightarrow S^{\prime}\right),(s, f) \mapsto \Sigma p: P(s) . P^{\prime}(f(p))\right)
\end{aligned}
$$

We then have

$$
\begin{aligned}
& \llbracket(S, P)+\left(S^{\prime}, P^{\prime}\right) \rrbracket_{\mathrm{cont}} \cong \llbracket S, P \rrbracket \mathrm{Cont}_{\mathrm{C}}+\llbracket S^{\prime}, P^{\prime} \rrbracket \mathrm{Cont}_{\mathrm{C}} \\
& \llbracket(S, P) \times\left(S^{\prime}, P^{\prime}\right) \rrbracket_{\mathrm{cont}} \cong \llbracket S, P \rrbracket_{\mathrm{Cont}} \times \llbracket S^{\prime}, P^{\prime} \rrbracket \text { Cont } \\
& \llbracket(S, P) \circ\left(S^{\prime}, P^{\prime}\right) \rrbracket \mathrm{Cont}_{\mathrm{C}} \cong \llbracket S, P \rrbracket \mathrm{Cont} \circ \llbracket S^{\prime}, P^{\prime} \rrbracket_{\mathrm{Cont}}
\end{aligned}
$$

Thus, the interpretation $(N)$ indeed restricts to the subcategory Cont:

Proposition 5.2. Define $(-)_{\text {Cont }}:$ Nest $\rightarrow$ Cont $\rightarrow$ Cont by

$$
\begin{array}{ll}
(\text { Id }) \text { Cont } C & =C \\
(K(S, P)) \text { Cont } C & =(S, P) \\
\left(\mathcal{F}_{0}+\mathcal{F}_{1}\right) \text { Cont } C & =\left(\mathcal{F}_{0}\right) \text { Cont } C+\left(\mathcal{F}_{1}\right) \text { Cont } C \\
\left(\mathcal{F}_{0} \times \mathcal{F}_{1}\right) \text { Cont } C & =\left(\mathcal{F}_{0}\right) \text { Cont } C \times\left(\mathcal{F}_{1}\right) \text { Cont } C \\
\left(\mathcal{F}_{0} \circledast \mathcal{F}_{1}\right) \text { Cont } C & =\left(\mathcal{F}_{0}\right) \text { Cont } C \circ\left(\mid \mathcal{F}_{1}\right) \text { Cont } C
\end{array}
$$

The following diagram then commutes:

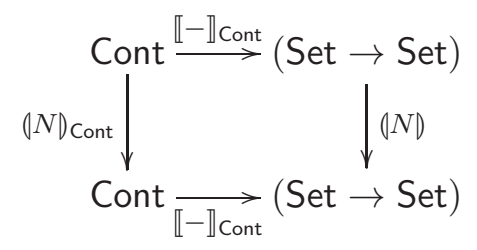


Coming back to our running example, if we consider the nested code $N_{L}$ for Lam, we have $\left(N_{L}\right)_{\text {Cont }}(S, P)=\left(S_{L}, P_{L}\right)$ with

$$
\begin{array}{ll}
S_{L} & =1+(S \times S)+\Sigma s: S \cdot P(s) \rightarrow 2 \\
P_{L}\left(\operatorname{in}_{1} *\right) & =1 \\
P_{L}\left(\operatorname{in}_{2}\left(s, s^{\prime}\right)\right) & =P(s)+P\left(s^{\prime}\right) \\
P_{L}\left(\operatorname{in}_{3}(s, f)\right) & =\Sigma p: P(s) \text {. if } f(p) \text { then } 1 \text { else } 0
\end{array}
$$

We see that indeed the positions $P$ show up in the equation for the shape $S_{L}$, so that we should expect an inductive-recursive definition to be the initial solution to this set of equations.

Nested Types are Containers. We know that Cont $=$ Fam $\left(\right.$ Set $\left.^{\mathrm{op}}\right)$. Now, we want to show that for every code $N$ : Nest, the functor $(N)$ Cont is a $\mathrm{IR}^{+}$functor: to see this one needs to carefully examine the constructions on families used to build $(N)$ cont. We will need to show that we can emulate the identity functor, containers and container product, coproduct and composition using $\mathrm{IR}^{+}$codes. Most of these are straightforward, but container composition will require some sophistication: we will need to observe that all $\mathrm{IR}^{+}$codes in question in fact are of a particularly simple, uniform form. We deal with each code in the nested grammar in turn.

Lemma $5.3\left(\mathrm{IR}^{+}\right.$codes for Id and $\left.K(S, P)\right)$.

(i) $\llbracket \delta_{1}\left(\left(X: 1 \rightarrow\right.\right.$ Set $\left.\left.^{\text {op }}\right) \mapsto \iota(X *)\right) \rrbracket C \cong C$.

(ii) $\llbracket \sigma_{S}(s \mapsto \iota P(s)) \rrbracket C \cong(S, P)$.

For encoding container coproducts, we can reuse the binary coproducts $+\mathrm{IR}$ on $\mathrm{IR}^{+}$ codes from Example 2.5.

Lemma $5.4\left(\mathrm{IR}^{+}\right.$codes for $\left.N+N^{\prime}\right)$. $\llbracket \gamma+\mathrm{IR} \gamma^{\prime} \rrbracket C \cong\left(\llbracket \gamma \rrbracket_{0} C+\llbracket \gamma^{\prime} \rrbracket_{0} C,\left[\llbracket \gamma \rrbracket_{1} C, \llbracket \gamma^{\prime} \rrbracket_{1} C\right]\right)$.

Emulating products of containers requires a little more work. The basic idea is that we get the product of two codes $\gamma$ and $\gamma^{\prime}$ by replacing all occurrences of the terminating code $\iota c$ in the first code $\gamma$ by the second code $\gamma^{\prime}$, where, in turn, we replace all codes $\iota c^{\prime}$ with $\iota\left(c \times c^{\prime}\right)$. In general, we can replace $\iota c^{\prime}$ with $\iota G\left(c, c^{\prime}\right)$ for a functor $G: \mathbb{C} \times \mathbb{C} \rightarrow \mathbb{C}$. Formally, we define a functor ${ }_{-}[\iota x \longmapsto \iota G(-, x)]: \mathrm{IR}^{+} \times \mathbb{C} \rightarrow \mathrm{IR}^{+}$for such a functor $G$ by

$$
\begin{aligned}
\left(\iota c^{\prime}\right)[\iota x \longmapsto \iota G(c, x)] & =\iota G\left(c, c^{\prime}\right) \\
\left(\sigma_{A} f\right)[\iota x \longmapsto G(c, x)] & =\sigma_{A}(\lambda a . f(a)[\iota x \longmapsto \iota G(c, x)]) \\
\left(\delta_{A} F\right)[\iota x \longmapsto G(c, x)] & =\delta_{A}(\lambda h . F(h)[\iota x \longmapsto \iota G(c, x)])
\end{aligned}
$$

See the formal development GMNF14 for the action on morphisms, which needs to be defined simultaneously in order to show that $F(h)[\iota x \longmapsto \iota G(c, x)]$ in the $\delta$ case again is a functor. Using this, we can now define the product $\gamma \times_{G} \gamma^{\prime}$ of two codes with respect to the functor $G$ :

$$
\begin{aligned}
(\iota c) \times_{G} \gamma & =\gamma[\iota x \longmapsto \iota G(c, x)] \\
\left(\sigma_{A} f\right) \times_{G} \gamma & =\sigma_{A}\left(\lambda a . f(a) \times_{G} \gamma\right) \\
\left(\delta_{A} F\right) \times_{G} \gamma & =\delta_{A}\left(\lambda h . F(h) \times_{G} \gamma\right)
\end{aligned}
$$

Again, we need to simultaneosuly show that $\times_{G}$ is functorial in order for $F(h) \times \gamma$ in the $\delta$ case to be a functor.

Lemma 5.5. $\llbracket \gamma \times_{G} \gamma^{\prime} \rrbracket C \cong\left(\llbracket \gamma \rrbracket_{0} C \times \llbracket \gamma^{\prime} \rrbracket_{0} C,\left(s, s^{\prime}\right) \mapsto G\left(\llbracket \gamma \rrbracket_{1} C s, \llbracket \gamma \rrbracket_{1} C s^{\prime}\right)\right)$. 
In particular, if we choose $G(X, Y)=X+Y$, we recover the container product.

Finally, we get to container composition. Composition of $\mathrm{IR}^{+}$codes (and IR codes) is an open problem in general, but since we are interested in emulating composition of containers, one could hope that there is more structure to be exploited, and this is indeed the case. The main insight is that all codes in the image of the translation are uniform, in the sense of unpublished work by Peter Hancock. Intuitively, a $\mathrm{IR}^{+}$code is uniform if the shape of the code (i.e. $\sigma / \delta / \iota$ followed by $\sigma / \delta / \iota$ followed by...) is independent of the arguments; e.g. $\sigma_{A}\left(\lambda x . \sigma_{B(x)}\left(\lambda y \cdot \delta_{C(x, y)}(\lambda z . \iota d(x, y, z))\right)\right)$ is uniform, while the code $\sigma_{\mathbb{N}}\left(\lambda x\right.$. if $x=17$ then $\delta_{B}(\lambda y \cdot \iota c(y))$ else $\sigma_{C(x)}\left(\lambda y \cdot \delta_{D(x, y)}(\lambda z . \iota d(x, y, z))\right.$ is not, since the shape is sometimes $\sigma-\delta-\iota$ and sometimes $\sigma-\sigma-\delta-\iota$. A precise description and study of uniform $\mathrm{IR}^{+}$codes is out of scope of this paper; for further information, we refer to our formal development GMNF14.

The main construction that uniform $\mathrm{IR}^{+}$codes allow, while arbitrary codes seem not to, is to construct a code for exponentiation of a $\mathrm{IR}^{+}$code $\gamma$ with a set $K$, i.e. a code $K \rightarrow \gamma$ such that $\llbracket K \rightarrow \gamma \rrbracket C \cong\left(K \rightarrow \llbracket \gamma \rrbracket_{0} C, f \mapsto \Sigma k: K . \llbracket \gamma \rrbracket_{1} C(f(k))\right)$. Note how there is a sigma type in the decoding of the family; as we have seen in Example 3.5, families closed under $\Sigma$ are canonical examples of a $\mathrm{IR}^{+}$construction. Since the construction of $K \rightarrow \gamma$ depends on the definition of uniform codes, we do not give it here, but refer again to our Agda formalisation GMNF14, where we also show that all constructions so far in this section result in uniform codes (except for the coproduct of codes, whose construction must be modified slightly). Given such a construction, we can now interpret also container composition with nested functors as a $\mathrm{IR}^{+}$code by defining $\bullet:$ Nest $\rightarrow \mathrm{IR}^{+} \rightarrow \mathrm{IR}^{+}$in the following way:

$$
\begin{aligned}
\mathrm{Id} \bullet \gamma & =\delta_{1}(\lambda X . X(*) \rightarrow \gamma) \\
K(S, P) \bullet \gamma & =\sigma_{S}(\lambda s . P(s) \rightarrow \gamma) \\
\left(N+N^{\prime}\right) \bullet \gamma & =(N \bullet \gamma)+\mathrm{IR}\left(N^{\prime} \bullet \gamma\right) \\
\left(N \times N^{\prime}\right) \bullet \gamma & =(N \bullet \gamma) \times_{+}\left(N^{\prime} \bullet \gamma\right) \\
\left(N \circledast N^{\prime}\right) \bullet \gamma & =N \bullet\left(N^{\prime} \bullet \gamma\right)
\end{aligned}
$$

Lemma 5.6. $\llbracket N \bullet \gamma \rrbracket C=\left((N) \mathrm{Cont}_{\mathrm{C}} C\right) \circ \llbracket \gamma \rrbracket C$.

Putting everything together, we arrive at the main theorem of this section.

Theorem 5.7. For every $N$ : Nest, the initial algebra $\mu(N)$ exists and is a container functor.

Proof. By Lemmas 5.3 to 5.6, $(N)$ cont is an $\mathrm{IR}^{+}$functor. Hence by the results in Section日, it has an initial algebra which is a container $\left(S_{N}, P_{N}\right)$. Since $\llbracket-\rrbracket$ Cont preserves initial objects and filtered colimits of cartesian morphisms (Abbott [Abb03], Propositions 4.5.1 and 4.6.7) and we know from Lemma 7.1 in Section 7 that the initial algebra chain of an $\mathrm{IR}^{+}$functor is made from cartesian morphisms only, we can conclude that $\llbracket\left(S_{N}, P_{N}\right) \rrbracket$ Cont $=\mu(N)$, showing that all nested types indeed are definable using containers.

\section{Comparison to Plain IR}

We now investigate the relationship between $\mathrm{IR}^{+}$and $\mathrm{IR}$. On the one hand we show in Proposition 6.1 how to embed Dybjer and Setzer's original coding scheme for IR into IR ${ }^{+}$; 
this way we can see IR as a subsystem of $\mathrm{IR}^{+}$. On the other hand we show in Proposition 6.2 that on discrete categories, the two schemas agrees having the same functorial interpretation; thus, using the canonical embedding of the discretisation of a category into itself, we can build a functor mapping $\mathrm{IR}^{+}$into IR.

Note that every type $D$ can be regarded as a discrete category, which we by abuse of notation denote $|D|$. In the other direction, every category $\mathbb{C}$ gives rise to a type $|\mathbb{C}|$ whose elements are the objects of $\mathbb{C}$.

Proposition 6.1. There is a function $\varphi: \operatorname{IR}(D) \rightarrow \mathrm{IR}^{+}|D|$ such that

$$
\llbracket \varphi(\gamma) \rrbracket_{\mathrm{IR}^{+}|D|}=\llbracket \gamma \rrbracket_{\mathrm{IR}(D)}
$$

Proof. The only interesting case is $\gamma=\delta_{A} F: \operatorname{IR}(D)$; we define $\varphi\left(\delta_{A} F\right)=\delta_{A}(\varphi \circ F)$. We need to ensure that $\varphi \circ F$ indeed is a functor, but since $|D|$ is a discrete, so is $A \rightarrow|D|$, and the mapping on objects $\varphi \circ F:(A \rightarrow|D|) \rightarrow \mathrm{IR}^{+}|D|$ can trivially be extended to a functor $(A \rightarrow|D|) \rightarrow \mathrm{IR}^{+}|D|$. It is easy to see that the two semantics do agree: on objects, the action is the same, and if ( $h$, id) is a morphism in Fam $|D|$, we see from the definition of $\llbracket \delta_{A} F \rrbracket_{\mathrm{IR}^{+}|D|}(h, k)$ in the proof of Theorem 3.3 that

$$
\begin{aligned}
\llbracket \delta_{A}(\varphi \circ F) \rrbracket_{\mathrm{IR}^{+}|D|}(h, \mathrm{id}) & =\left[\mathrm{in}_{h \circ g} \circ \llbracket \varphi\left(F(Q \circ h \circ g) \rrbracket(h, \mathrm{id}) \circ \llbracket(\varphi \circ F) \rightarrow\left(g^{*}(\mathrm{id})\right) \rrbracket_{(X, P)}\right]_{g: A \rightarrow X}\right. \\
& =\left[\mathrm{in}_{h \circ g} \circ \llbracket \varphi(F(Q \circ h \circ g) \rrbracket(h, \mathrm{id})]_{g: A \rightarrow X}\right. \\
& =\left[\mathrm{in}_{h \circ g} \circ \llbracket F(Q \circ h \circ g) \rrbracket_{\operatorname{IR}(D)}(h, \mathrm{id})\right]_{g: A \rightarrow X} \\
& =\llbracket \delta_{A} F \rrbracket_{\mathrm{IR}(D)}
\end{aligned}
$$

where $\llbracket(\varphi \circ F)_{\rightarrow}\left(g^{*}(\mathrm{id})\right) \rrbracket_{(X, P)}=\mathrm{id}$ since $\llbracket(\varphi \circ F)(-) \rrbracket_{(X, P)}$ is a functor.

This proposition shows that the theory of IR can be embedded in the theory of $\mathrm{IR}^{+}$. Some readers might perhaps be surprised that we only define a function $\operatorname{IR}(D) \rightarrow \operatorname{IR}^{+}|D|$, and not a functor. The reason is the mismatch of morphisms between $\operatorname{IR}(D)$ and $\operatorname{IR}^{+}|D|$; because $\operatorname{IR}(D)$ has a full and faithful embedding into Fam $|D| \rightarrow$ Fam $|D|$, whereas $\operatorname{IR}^{+}|D|$ has not, there are necessarily morphisms in $\operatorname{IR}(D)$ that have no counterpart in $\operatorname{IR}^{+}|D|$. Going the other way, we are more successful, and can make the previous result more precise: using the functoriality of Fam (Remarks 2.3), we can embed Fam $|\mathbb{C}|$ into $\operatorname{Fam}(\mathbb{C})$. We can then show that forgetting about the extra structure of $\mathbb{C}$ in $I R^{+}$simply gets us back to plain IR.

Proposition 6.2. Let $\varepsilon:|\mathbb{C}| \rightarrow \mathbb{C}$ the canonical embedding of the discretisation of a category $\mathbb{C}$ into itself. There is a functor $\psi:\left|\mathbb{R}^{+} \mathbb{C} \rightarrow\right| \mathbb{R}|\mathbb{C}|$ such that for all $\gamma: \mathbb{R}^{+} \mathbb{C}$

$$
\operatorname{Fam}(\varepsilon) \circ \llbracket \psi(\gamma) \rrbracket_{|\mathrm{R}| \mathbb{C} \mid} \cong \llbracket \gamma \rrbracket_{\mathrm{IR}^{+}(\mathbb{C})} \circ \operatorname{Fam}(\varepsilon)
$$

Furthermore, $\psi \circ \varphi=\mathrm{id}$, where $\varphi: \operatorname{IR}(\mathbb{C}) \rightarrow \mathrm{IR}^{+}|\mathbb{C}|$ is the function from Proposition 6.1.

Proof. We define the functor $\psi: \mathbb{I}^{+}(\mathbb{C}) \rightarrow \mathbb{I R}|\mathbb{C}|$ by recursion on the structure of $\gamma$. On objects, $\psi$ is defined as follows:

$$
\begin{aligned}
\psi(\iota c) & =\iota c \\
\psi\left(\sigma_{A} f\right) & =\sigma_{A}(a \mapsto \psi(f a)) \\
\psi\left(\delta_{A} F\right) & =\delta_{A}(X \mapsto \psi(F(\varepsilon \circ X)))
\end{aligned}
$$

We now use full and faithfulness of the interpretation functor $\llbracket-\rrbracket_{|R| \mathbb{C} \mid}$, as proved in Ghani et al. $\mathrm{GHM}^{+} 13$, to let the function $\psi$ act on morphisms as well as on objects. Since the two 
interpretation functors agree on objects, i.e. $\llbracket \gamma \rrbracket(X, P)=\llbracket \psi(\gamma) \rrbracket_{\mathrm{IR}|\mathbb{C}|}(X, P)$, a $\mathrm{IR}^{+}$morphism $\rho: \gamma \rightarrow \gamma^{\prime}$ corresponds to a natural transformation $\llbracket \rho \rrbracket: \llbracket \psi(\gamma) \rrbracket_{\mathbb{I R}|\mathbb{C}|} \rightarrow \llbracket \psi\left(\gamma^{\prime}\right) \rrbracket_{|\mathbb{R}| \mathbb{C} \mid}$. By full and faithfulness of $\llbracket-\rrbracket_{|R| \mathbb{C} \mid}$ such a natural transformation corresponds to an IR morphism $\psi(\gamma) \rightarrow \psi\left(\gamma^{\prime}\right)$ which we take as the definition of $\psi(\rho)$. Similarly, full and faithfulness of $\llbracket-\rrbracket|\mathbb{R}| \mathbb{C} \mid$ ensure that composition and identity are preserved by $\psi$, which is therefore a functor.

We are left with checking that (因) holds for morphisms. Recall from Remarks 2.3 that a morphism in Fam $|\mathbb{C}|$ correspond to a split cartesian morphism in $\operatorname{Fam}(\mathbb{C})$, i.e one whose second component is an identity. Thus, to verify (因) it is enough to check that $\llbracket \gamma \rrbracket$ preserves such split cartesian morphisms. The interesting case is $\gamma=\delta_{A} F$. Let $(h$, id $):(X, P \circ h) \rightarrow$ $(Y, P)$ be a morphism in Fam $|\mathbb{C}|$. We have

$$
\begin{aligned}
\llbracket \delta_{A} F \rrbracket(h, \text { id }) & =\left[i n_{h \circ g} \circ \llbracket F(P \circ h \circ g) \rrbracket(h, \text { id }) \circ \llbracket F_{\rightarrow}\left(g^{*}(\text { id })\right) \rrbracket_{(X, P)}\right]_{g: A \rightarrow X} \\
& =\left[i n_{h \circ g} \circ \llbracket F(P \circ h \circ g) \rrbracket(h, \text { id })\right]_{g: A \rightarrow X}
\end{aligned}
$$

where $\llbracket F\left(g^{*}\right.$ id $) \rrbracket_{(X, P)}=$ id since $g^{*}, F$ and $\llbracket-\rrbracket$ are functors. By the induction hypothesis, each $\llbracket F(P \circ h \circ g) \rrbracket(h$, id $)$ is split cartesian. Furthermore injections are split cartesian in Fam $(\mathbb{C})$, and since compositions and cotuplings of split cartesian morphisms are still split cartesian in $\operatorname{Fam}(\mathbb{C})$ we conclude that $\llbracket \delta_{A} F \rrbracket(h$, id) indeed is a split cartesian morphism as required.

Finally, since $\varepsilon$ is the identity on discrete categories the two schemas agrees on discrete categories and we automatically get $\psi \circ \varphi=$ id.

\section{Existence of Initial Algebras}

We briefly revisit the initial algebra argument used by Dybjer and Setzer [DS99. Inspecting their proof, we see that it indeed is possible to adapt it also for the more general setting of positive inductive-recursive definitions by making the appropriate adjustments.

Remember that a morphism $(h, k):(U, T) \rightarrow\left(U, T^{\prime}\right)$ in $\operatorname{Fam}(\mathbb{C})$ is a split cartesian morphism if $k=\mathrm{id}_{T}$, i.e. $T^{\prime} \circ h=T$, and that Fam $|\mathbb{C}|$ is the subcategory (subfibration) of Fam $(\mathbb{C})$ with the same objects, but with morphisms the split cartesian ones only. The proof of existence of initial algebras for IR functors as given by Dybjer and Setzer [DS99] takes place in the category Fam $|\mathbb{C}|$. The hard work of the proof is divided between two lemmas. First Dybjer and Setzer prove that an IR functor $\llbracket \gamma \rrbracket$ preserves $\kappa$-filtered colimits if $\kappa$ is an inaccessible cardinal which suitably bounds the size of the index sets in the image of the filtered diagram. Secondly they use the assumption of the existence of a large cardinal, namely a Mahlo cardinal, to prove that such a cardinal bound for the index sets can actually be found. The exact definition of when a cardinal is a Mahlo cardinal will not be important for the current presentation; see Dybjer and Setzer [DS99], or the second author's thesis [Mal15] for how this assumption is used. The existence of an initial algebra then follows a standard argument: the initial algebra of a $\kappa$-continuous functor can be constructed as the colimit of the initial chain up to $\kappa$ iterations (see e.g. Adámek et al. AMM10]).

Inspecting the proofs, we see that they crucially depend on morphisms being split cartesian in several places. Luckily, the morphisms involved in the corresponding proofs for $\mathrm{IR}^{+}$actually are! As is well-known, a weaker condition than $\kappa$-continuity is actually sufficient: it is enough that the functor in question preserve the specific colimit of the initial 
$\kappa$-chain. We thus show that the initial chain of a $\mathrm{IR}^{+}$functor actually lives in $\mathrm{Fam}|\mathbb{C}|$, which will allow us to modify Dybjer and Setzer's proof accordingly.

Lemma 7.1. For each $\gamma: \mathbb{I}^{+} \mathbb{C}$, the initial chain

$$
0 \rightarrow \llbracket \gamma \rrbracket(0) \rightarrow \llbracket \gamma \rrbracket^{2}(0) \rightarrow \ldots
$$

consists of split cartesian morphisms only.

Proof. Recall that the connecting morphisms $\omega_{j, k}: \llbracket \gamma \rrbracket^{j}(0) \rightarrow \llbracket \gamma \rrbracket^{k}(0)$ are uniquely determined as follows:

- $\omega_{0,1}=!_{\llbracket \gamma \rrbracket(0)}$ is unique.

- $\omega_{j+1, k+1}$ is $\llbracket \gamma \rrbracket\left(\omega_{j, k}\right): \llbracket \gamma \rrbracket\left(\llbracket \gamma \rrbracket^{j}(0)\right) \rightarrow \llbracket \gamma \rrbracket\left(\llbracket \gamma \rrbracket^{k}(0)\right)$.

- $\omega_{j, k}$ is the colimit cocone for $j$ a limit ordinal.

We prove the statement by induction on $j$. It is certainly true that $! \llbracket \gamma(0):(0, !) \rightarrow \llbracket \gamma \rrbracket(0)$ is an identity at each component - there are none. Thus $\omega_{0,1}$ is a split cartesian morphism. At successor stages, we apply Proposition 6.2 and the induction hypothesis. Finally, at limit stages, we use the fact that the colimit lives in Fam $|\mathbb{C}|$ and hence coincides with the colimit in that category on split cartesian morphisms, so that the colimit cocone is split cartesian.

Inspecting Dybjer and Setzer's original proof, we see that it now goes through also for $\mathrm{IR}^{+}$if we insert appeals to Lemma 7.1 where necessary. To finish the proof, we also need to ensure that $\operatorname{Fam}(\mathbb{C})$ has $\kappa$-filtered colimits; this is automatically true if $\mathbb{C}$ has all small connected colimits (compare Remarks 2.3), since $\operatorname{Fam}(\mathbb{C})$ then is cocomplete. Note that discrete categories have all small connected colimits for trivial reasons.

Theorem 7.2. Assume that a Mahlo cardinal exists in the meta-theory. If $\mathbb{C}$ has connected colimits, then every functor $\llbracket \gamma \rrbracket$ for $\gamma: \mathbb{I R}^{+} \mathbb{C}$ has an initial algebra.

\section{Conclusions and Future Work}

In this paper we have introduced the theory $\mathrm{IR}^{+}$of positive inductive-recursive definitions as a generalization of Dybjer and Setzer's theory IR of inductive-recursive definitions [DS99, DS03, DS06], different from the fibrational generalization explored in Ghani et al. GMNFS13: by modifying both syntax and semantics of IR we have been able to broaden the semantics to all of $\operatorname{Fam}(\mathbb{C})$ and not just Fam $|\mathbb{C}|$. The theory of $\mid R^{+}$, with $\mid R$ as a subtheory, paves the way to the analysis of more sophisticated data types which allow not only for the simultaneous definition of an inductive type $X$ and of a recursive function $f: X \rightarrow D$, but also takes the intrinsic structure between objects in the target type $D$ into account. This is the case for example when $D$ is a setoid, the category Set or Set ${ }^{\mathrm{op}}$, a groupoid or, even more generally, an arbitrary category $\mathbb{C}$.

In future work we aim to explore the theory of $\mathrm{IR}^{+}$from a fibrational perspective: this will allow us to reconcile the theory of $\mathrm{IR}^{+}$with the analysis of $I R$ as given in Ghani et al. GMNFS13. In particular this will amount to characterising the semantics of $\delta$ codes as left Kan extensions. An open problem for both $\mathrm{IR}^{+}$and IR is the question whether the definable functors are closed under composition, i.e. if there is a code $\gamma \circ \gamma^{\prime}$ such that $\llbracket \gamma \circ \gamma^{\prime} \rrbracket \cong \llbracket \gamma \rrbracket \circ \llbracket \gamma^{\prime} \rrbracket$ for all codes $\gamma$ and $\gamma^{\prime}$. Another interesting direction of research is to investigate to which extent the rich structure of the families construction Fam will help 
shed light on the analysis of $\mathrm{IR}^{+}$types: in particular to exploit the monadic structure of Fam and then to investigate the relationship between the theory of $\mathrm{IR}^{+}$and the theory of familial 2-functors introduced by Weber Web07.

\section{REFERENCES}

[AAG05] Michael Abbott, Thorsten Altenkirch, and Neil Ghani. Containers: Constructing strictly positive types. Theoretical Computer Science, 342(1):3 - 27, 2005.

[Abb03] Michael Abbott. Category of Containers. PhD thesis, University of Leicester, 2003.

[Acz80] Peter Aczel. Frege structures and the notions of proposition, truth and set. In Jon Barwise, H. Jerome Keisler, and Kenneth Kunen, editors, The Kleene Symposium, volume 101 of Studies in Logic and the Foundations of Mathematics, pages 31 - 59. Elsevier, 1980.

[AMM10] Jiri Adámek, Stefan Milius, and Lawrence Moss. Initial algebras and terminal coalgebras: a survey. Draft, June 292010.

[AMU05] Andreas Abel, Ralph Matthes, and Tarmo Uustalu. Iteration and coiteration schemes for higherorder and nested datatypes. Theoretical Computer Science, 333(1-2):3-66, 2005.

[Bla00] Paul Blampied. Structured Recursion for Non-uniform Data-types. PhD thesis, University of Nottingham, 2000.

[CJ95] Aurelio Carboni and Peter Johnstone. Connected limits, familial representability and Artin glueing. Mathematical Structures in Computer Science, 5(04):441-459, 1995.

[DS99] Peter Dybjer and Anton Setzer. A finite axiomatization of inductive-recursive definitions. In Typed lambda calculi and applications: 4th international conference, TLCA'99, L'Aquila, Italy, April 7-9, 1999: proceedings, pages 129-146. Springer Verlag, 1999.

[DS03] Peter Dybjer and Anton Setzer. Induction-recursion and initial algebras. Annals of Pure and Applied Logic, 124(1-3):1-47, 2003.

[DS06] Peter Dybjer and Anton Setzer. Indexed induction-recursion. Journal of logic and algebraic programming, 66(1):1-49, 2006.

[Dyb00] Peter Dybjer. A general formulation of simultaneous inductive-recursive definitions in type theory. Journal of Symbolic Logic, 65(2):525-549, 2000.

[EHA09] Linus Ek, Ola Holmström, and Stevan Andjelkovic. Formalizing Arne Andersson trees and Leftleaning Red-Black trees in Agda. Bachelor thesis, Chalmers University of Technology, 2009.

[FPT99] Marcelo Fiore, Gordon Plotkin, and Daniele Turi. Abstract syntax and variable binding. In Proc. Logic in Computer Science, pages 193-202, 1999.

$\left[\mathrm{GHM}^{+} 13\right]$ Neil Ghani, Peter Hancock, Lorenzo Malatesta, Conor McBride, and Thorsten Altenkirch. Small induction recursion. In TLCA 2013, 2013.

[GHUV06] Neil Ghani, Makoto Hamana, Tarmo Uustalu, and Varmo Vene. Representing cyclic structures as nested types. Presented at Trends in Functional Programming, 2006.

[GMNF13] Neil Ghani, Lorenzo Malatesta, and Fredrik Nordvall Forsberg. Positive inductive-recursive definitions. In CALCO 2013, 2013.

[GMNF14] Neil Ghani, Lorenzo Malatesta, and Fredrik Nordvall Forsberg. Positive inductive-recursive definitions: Agda formalisation, 2014. https://personal.cis.strath.ac.uk/fredrik.nordvall-forsberg/positive_IR/

[GMNFS13] Neil Ghani, Lorenzo Malatesta, Fredrik Nordvall Forsberg, and Anton Setzer. Fibred data types. In LICS 2013, 2013.

[Hin00] Ralf Hinze. Functional pearl: Perfect trees and bit-reversal permutation. Journal of Functional Programming, 10(3):305-317, 2000.

[Jac99] Bart Jacobs. Categorical Logic and Type Theory, volume 141 of Studies in Logic and the Foundations of Mathematics. North Holland, Elsevier, 1999.

[Mal15] Lorenzo Malatesta. Investigations into Inductive-Recursive Definitions. PhD thesis, University of Strathclyde, 2015.

[ML72] Per Martin-Löf. An intuitionistic theory of types. Published in Twenty-Five Years of Constructive Type Theory, 1972.

[ML84] Per Martin-Löf. Intuitionistic type theory. Bibliopolis Naples, 1984. 
[MM04] Conor McBride and James McKinna. The view from the left. Journal of Functional Programming, 14(1):69-111, 2004.

[NF13] Fredrik Nordvall Forsberg. Inductive-inductive definitions. PhD thesis, Swansea University, 2013.

[NFS12] Fredrik Nordvall Forsberg and Anton Setzer. A finite axiomatisation of inductive-inductive definitions. In Ulrich Berger, Diener Hannes, Peter Schuster, and Monika Seisenberger, editors, Logic, Construction, Computation, volume 3 of Ontos mathematical logic, pages $259-287$. Ontos Verlag, 2012.

[Uni13] The Univalent Foundations Program. Homotopy Type Theory: Univalent Foundations of Mathematics. http://homotopytypetheory.org/book, Institute for Advanced Study, 2013.

[Web07] Mark Weber. Familial 2-functors and parametric right adjoints. Theory and Applications of Category Theory, 18(22):665-732, 2007. 